\title{
On the Accuracy of Isosurfaces in Tomographic Volume Visualization
}

\author{
Andreas Pommert, Ulf Tiede, and Karl Heinz Höhne \\ Institute of Mathematics and Computer Science in Medicine (IMDM) \\ University Hospital Hamburg-Eppendorf, 20251 Hamburg, Germany \\ \{pommert, tiede, hoehne\}@uke.uni-hamburg.de
}

\begin{abstract}
Results of tomographic volume visualization depend on a large number of acquisition and processing parameters. In this study, localization accuracy of isosurfaces is investigated, using simulated and phantom data. Guidelines for choosing parameters are given, and a pictorial index of typical effects resulting from various parameter settings is presented. Provided that a suitable threshold value is used, it is shown that the accuracy is almost one order of magnitude better than the scanner resolution.
\end{abstract}

\section{Introduction}

Visualization of volume data obtained in computer tomography (CT) or magnetic resonance imaging (MRI) is an important aid for diagnosis, treatment planning, surgery rehearsal, education, and research [5]. For clinical applications, it is of course important to assure that the 3D images really show the true anatomical situation, or at least to know about their limitations. Unfortunately, the resulting images depend on a large number of parameters, including pixel size, convolution kernel of the scanner, slice distance and thickness, interpolation method, sampling distance, and threshold value (or other segmentation parameters). Variation of these parameters may result in very different images.

While image fidelity has been identified as a major research topic in volume visualization, not many investigations on this subject are available to date [4]. In this paper, we are investigating the accuracy of the visualization of isointensity surfaces (or isosurfaces, for short).

\section{Materials and Methods}

\subsection{Volume Visualization}

Volume visualization is performed using a ray casting approach. For interpolation of the intensities at the sampling points, linear interpolation, quadratic interpolation [2], or cubic splines (B-spline, Catmull-Rom spline) are applied [3]. Between the sampling points, an isosurface is located with a bisection algorithm. Details of the visualization algorithms may be found in [7]. 
High contrast objects such as bone are often segmented using intensity thresholds. These are mostly interactively set, considering e.g. smoothness of the resulting surface, appearance of artifacts, or choosing the $50 \%$ value between approximate background and object intensities. To get somewhat more reproducible results, a threshold may be selected such that the integrated gradient magnitude over all surface points is maximized, as proposed in the contour spectrum approach [1]. Results of segmentation are represented by object membership labels assigned to every voxel.

\subsection{Evaluation}

Simulated Data. Using simulated data for the evaluation of medical volume visualization was first proposed in [6]. In this study, we use a numerically described test scene, from which tomographic volume data are generated with an oversampling and postfiltering approach [8]. This way, the partial volume effect can be modeled realistically. Neutral, smoothing and edge enhancing kernels are simulated, using Catmull-Rom spline, Gaussian, and BC-spline with $B=0, C=1$ [3], respectively.

The test scene of $32 \times 32 \times 32$ (virtual) $\mathrm{mm}^{3}$ is a three-dimensional extension of the siemens star, as used in photography, e.g. (fig. 1 left). The star has a radius of $10 \mathrm{~mm}$, and the 12 cones have a maximum radius of $1.3 \mathrm{~mm}$ each, giving equal size of cones and spaces in between. It is slightly tilted to avoid alignment with the voxel grid. This scene is especially suitable to simulate small structures. Object and background intensities are set to 2000 and 1000, respectively. Furthermore, (rather strong) non-correlated gaussian noise $(\sigma=100)$ may be added. The white ring shown on the images is indicating positions with a cone diameter of $1.0 \mathrm{~mm}$.

In order to measure the accuracy of an isosurface, the localization error, i.e. the difference between actual and ideal surface position (obtained from the numerical description) is calculated along each viewing ray. Since it may be positive or negative, the localization error for a whole image is calculated as the mean absolute localization error.
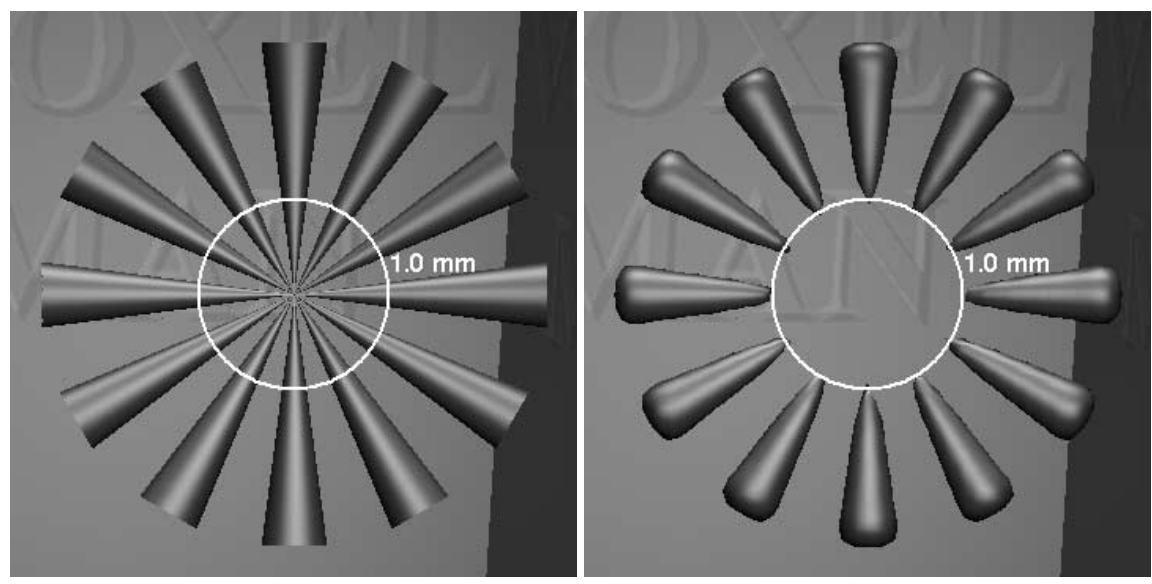

Fig. 1. The test object rendered from its mathematical definition (left), and from simulated volume data, using the basic configuration of acquisition and visualization parameters (right). 
Phantom Data. For verification of the results on real tomographic data, a teflon cone (height $30.0 \mathrm{~mm}$, diameter $10.0 \mathrm{~mm}$ ) is used which was scanned on a Siemens Somatom Plus 4 CT scanner (voltage $120 \mathrm{kV}$, exposure $180 \mathrm{mAs}$, 87 slices, pixel size $0.098 \mathrm{~mm}$, convolution kernel SP90, slice distance $0.5 \mathrm{~mm}$, slice thickness $1.0 \mathrm{~mm}$ ). A Catmull-Rom spline interpolation was performed to obtain isotropic voxels. A numerical description of the cone was created and interactively matched to the data to allow measurements of localization accuracy.

\section{Results}

An exploration of the whole parameter space is hardly feasible. Therefore, a reasonable basic configuration of parameters is selected and later modified. Measurements of the localization error are listed in tab. 1. Since the best threshold value is usually not known, errors are given for the $50 \%$ value of 1500 and the somewhat extreme settings of 1200 and 1800 , respectively.

Table 1. Measurements of the localization error for the simulated data (mean absolute error \pm standard deviation). The ${ }^{*}$ denotes the basic configuration of acquisition and processing parameters used in this study.

\begin{tabular}{|c|c|c|c|c|}
\hline & \multicolumn{3}{|c|}{ threshold value } \\
\hline & & 1200 & 1500 & 1800 \\
\hline varied parameter & value & \multicolumn{3}{|c|}{ localization error $[\mathrm{mm}]$} \\
\hline \multirow[t]{2}{*}{ gaussian noise, $\sigma$} & 0 & $0.32 \pm 0.12$ & $0.12 \pm 0.11$ & $0.47 \pm 0.12$ \\
\hline & $100^{*}$ & $0.56 \pm 1.51$ & $0.13 \pm 0.13$ & $0.47 \pm 0.15$ \\
\hline \multirow[t]{3}{*}{ voxel size } & $0.25 \mathrm{~mm}$ & $0.85 \pm 2.34$ & $0.11 \pm 0.12$ & $0.44 \pm 0.16$ \\
\hline & $0.5 \mathrm{~mm}^{*}$ & $0.56 \pm 1.51$ & $0.13 \pm 0.13$ & $0.47 \pm 0.15$ \\
\hline & $1.0 \mathrm{~mm}$ & $0.45 \pm 0.67$ & $0.23 \pm 0.18$ & $0.66 \pm 0.19$ \\
\hline \multirow[t]{3}{*}{ resolution } & $0.25 \mathrm{~mm}$ & $0.44 \pm 1.49$ & $0.08 \pm 0.10$ & $0.28 \pm 0.17$ \\
\hline & $0.5 \mathrm{~mm}$ & $0.46 \pm 1.50$ & $0.08 \pm 0.09$ & $0.32 \pm 0.14$ \\
\hline & $1.0 \mathrm{~mm}^{*}$ & $0.56 \pm 1.51$ & $0.13 \pm 0.13$ & $0.47 \pm 0.15$ \\
\hline \multirow[t]{3}{*}{ convolution kernel } & smoothing & $0.57 \pm 1.53$ & $0.22 \pm 0.17$ & $0.66 \pm 0.18$ \\
\hline & neutral $^{*}$ & $0.56 \pm 1.51$ & $0.13 \pm 0.13$ & $0.47 \pm 0.15$ \\
\hline & edge enhancing & $0.57 \pm 1.51$ & $0.09 \pm 0.13$ & $0.38 \pm 0.15$ \\
\hline \multirow[t]{3}{*}{ anisotropic data } & SL $1 \mathrm{~mm}$ & $0.56 \pm 1.51$ & $0.13 \pm 0.13$ & $0.48 \pm 0.15$ \\
\hline & SL $2 \mathrm{~mm}$ & $0.54 \pm 1.54$ & $0.20 \pm 0.16$ & $0.58 \pm 0.17$ \\
\hline & SL $1 \mathrm{~mm}$, PI $2 \mathrm{~mm}$ & $0.55 \pm 1.52$ & $0.18 \pm 0.15$ & $0.55 \pm 0.16$ \\
\hline \multirow[t]{5}{*}{ sampling distance } & 0.25 voxels & $0.67 \pm 1.80$ & $0.13 \pm 0.14$ & $0.47 \pm 0.16$ \\
\hline & 0.5 voxels ${ }^{*}$ & $0.56 \pm 1.51$ & $0.13 \pm 0.13$ & $0.47 \pm 0.15$ \\
\hline & 1.0 voxels & $0.47 \pm 1.18$ & $0.13 \pm 0.13$ & $0.45 \pm 0.14$ \\
\hline & 2.0 voxels & $0.39 \pm 0.76$ & $0.12 \pm 0.12$ & $0.44 \pm 0.12$ \\
\hline & 4.0 voxels & $0.38 \pm 0.69$ & $0.11 \pm 0.11$ & $0.43 \pm 0.12$ \\
\hline \multirow[t]{5}{*}{ interpolation } & linear* & $0.56 \pm 1.51$ & $0.13 \pm 0.13$ & $0.47 \pm 0.15$ \\
\hline & quadratic, $r=0.5$ & $0.33 \pm 0.13$ & $0.14 \pm 0.13$ & $0.49 \pm 0.13$ \\
\hline & quadratic, $r=1.0$ & $1.53 \pm 3.23$ & $0.13 \pm 0.13$ & $0.44 \pm 0.17$ \\
\hline & B-spline & $0.34 \pm 0.13$ & $0.15 \pm 0.13$ & $0.52 \pm 0.13$ \\
\hline & Catmull-Rom & $1.75 \pm 3.57$ & $0.12 \pm 0.13$ & $0.41 \pm 0.16$ \\
\hline
\end{tabular}




\subsection{Basic Configuration of Parameters}

The basic limiting factor of tomographic image acquisition is the resolution of the scanner, which is determined (among others) by the convolution kernel. In this study, it is arbitrarily set to $1.0 \mathrm{~mm}$. According to the sampling theorem, the resulting signal has to be sampled with at least two samples per $\mathrm{mm}$ to avoid aliasing effects. Voxel size is thus set to $0.5 \mathrm{~mm}$.

For resampling on the ray, a sampling distance of 1 voxel might seem sufficient; however, if the volume is rotated, intensity changes might be as close as $1 / \sqrt{3}$ voxels. Therefore, the sampling distance is set to 0.5 voxels.

Except for a very low threshold, there are only small differences between noiseless and noisy data. Therefore, all further measurements of the localization error are based on the noisy data. However, to make the effects more apparent, the images shown are rendered from the noiseless data.

\subsection{Variation of Image Acquisition Parameters}

Voxel Size With a smaller voxel size of $0.25 \mathrm{~mm}$ (fig. 2] top left), resolution is only slightly increased, as can be seen at the tips of the cones, at a price of using much more data. Improvement of the localization error is equally small. Vice versa, with the voxel size enlarged to $1 \mathrm{~mm}$, the test object is strongly deformed (fig. 2, top right).

Scanner Resolution With the resolution of the scanner convolution kernel improved to $0.25 \mathrm{~mm}$ or $0.5 \mathrm{~mm}$ (fig. 2, middle row), the tips of the cones are extended, and the localization error is improved. However, since the voxel size is now becoming the limiting factor, some noticeable aliasing occurs, especially for the smaller resolution.

Convolution Kernel Using an edge enhancing instead of a neutral scanner convolution kernel, the localization error decreases when using a suitable threshold. This is not really surprising, considering our small test object. Vice versa, results are getting worse with a smoothing kernel.

Anisotropic Data If a box shaped slice sensitivity profile is used in axial direction (top to bottom on the images), little is changed for a slice thickness (SL) of $1 \mathrm{~mm}$. However, for a slice thickness of $2 \mathrm{~mm}$, the anisotropic resolution is clearly visible (fig. 2 bottom left). The localization error also deteriorates. Somewhat better results are obtained with a simulated spiral CT with a slice thickness of $1 \mathrm{~mm}$ and a pitch (PI) of 2 (fig. 2 bottom right).

\subsection{Variation of Visualization Parameters}

Interpolation Compared to linear interpolation, the Catmull-Rom spline gives a slightly improved resolution, while the smoothing B-spline makes it worse (fig. 3 top row). Surprisingly, localization errors are not improved for the Catmull-Rom spline, due to its higher sensitivity to noise. Quadratic interpolation methods with $q=0.5$ and $q=1.0$ perform comparably to B-spline and Catmull-Rom spline, respectively.

Sampling Distance Increase to 1 or 2 voxels creates barely visible or noticable undersampling artifacts, respectively (fig. 3. middle row). Surprisingly, the localization error 

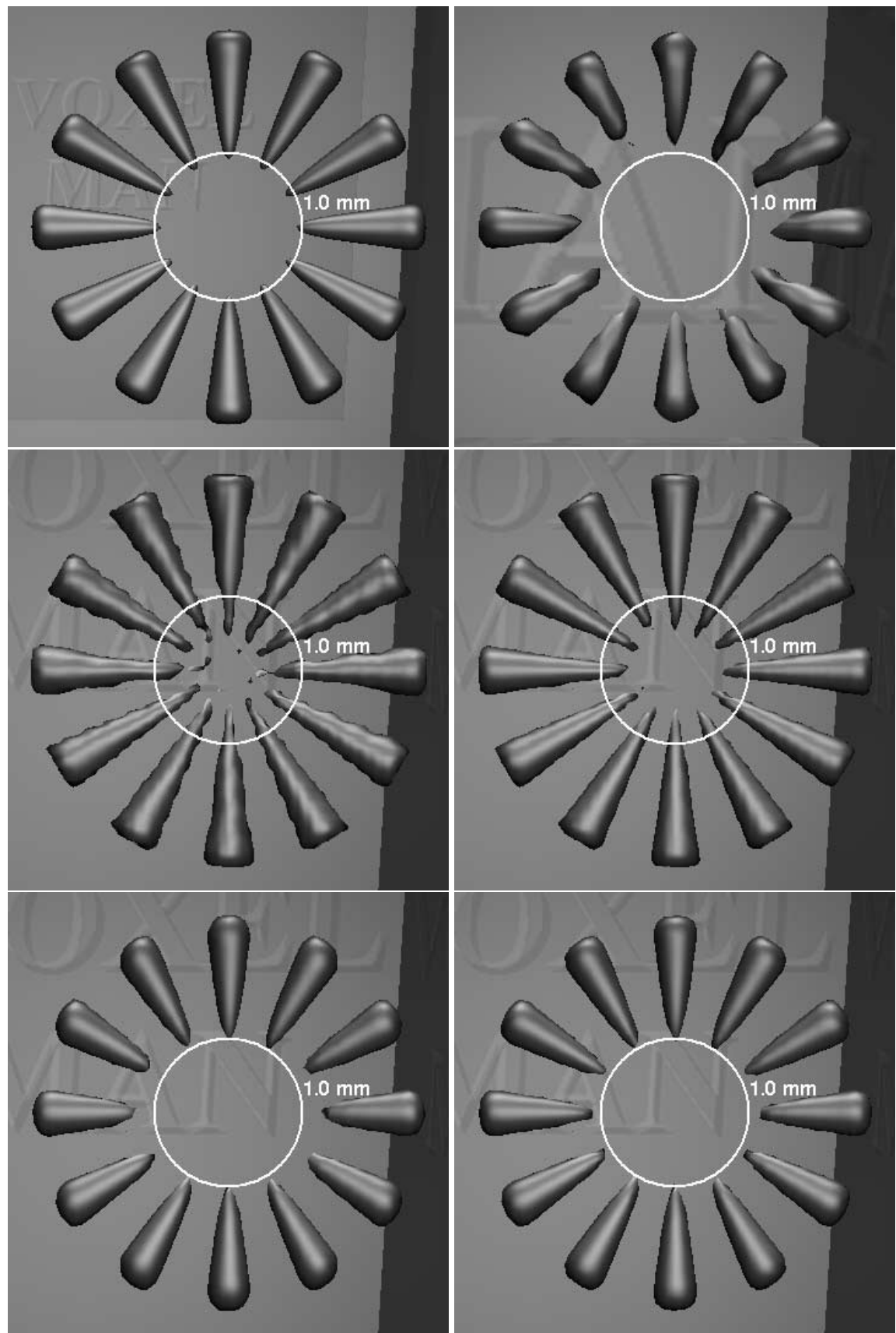

Fig. 2. Variation of tomographic acquisition parameters. Top: pixel size $0.25 \mathrm{~mm}$ (left) and $1.0 \mathrm{~mm}$ (right). Middle: increased scanner resolution of $0.25 \mathrm{~mm}$ (left) and $0.5 \mathrm{~mm}$ (right). Bottom: anisotropic data, with a slice thickness of $2 \mathrm{~mm}$ (left), and spiral acquisition with a slice thickness of $1 \mathrm{~mm}$ and a pitch of 2 (right). 

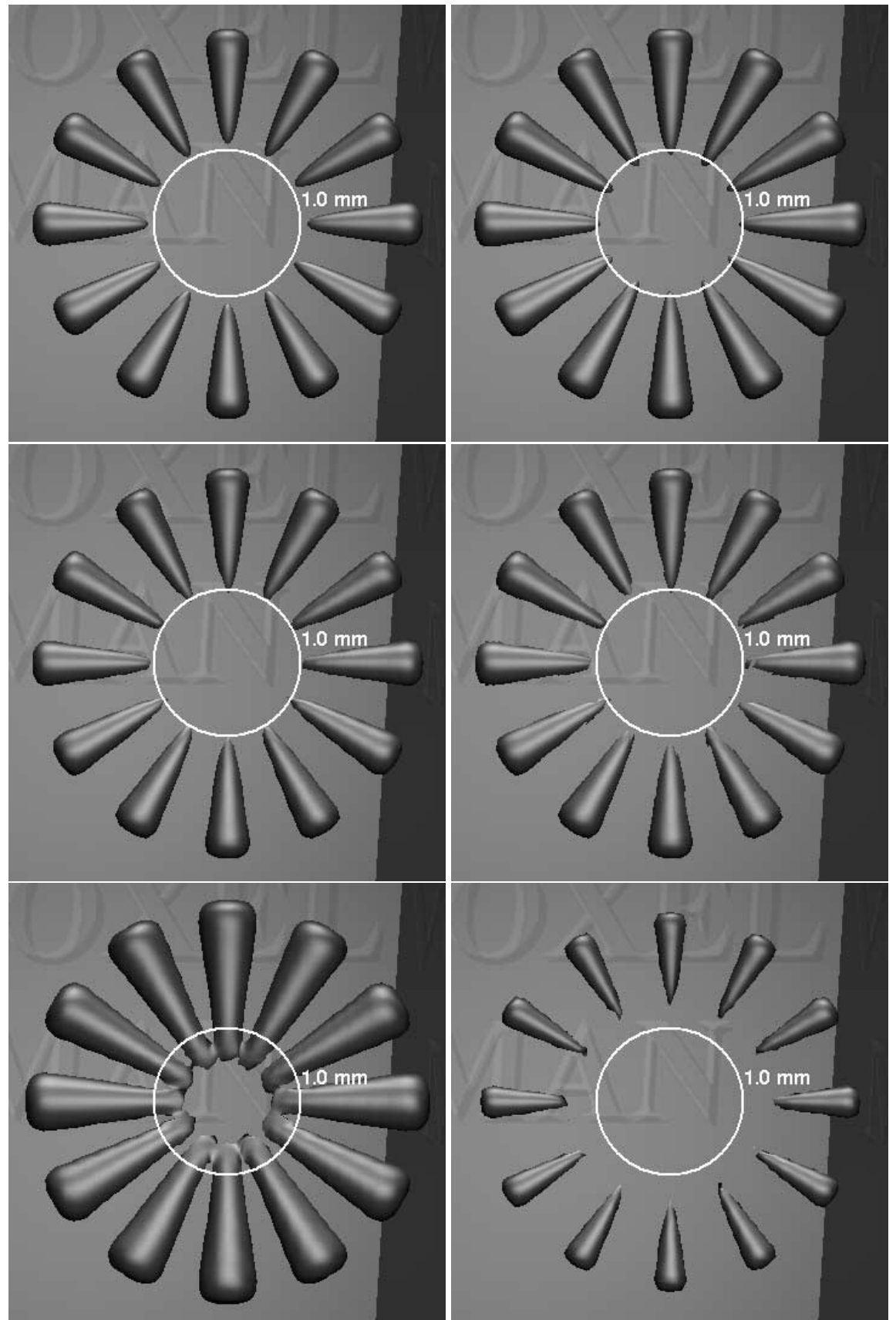

Fig. 3. Variation of visualization and segmentation parameters. Top: interpolation using B-spline (left) and Catmull-Rom spline (right). Middle: increased sampling distance of 1 voxel (left) and 2 voxels (right). Bottom: threshold value of 1200 (left) and 1800 (right). 

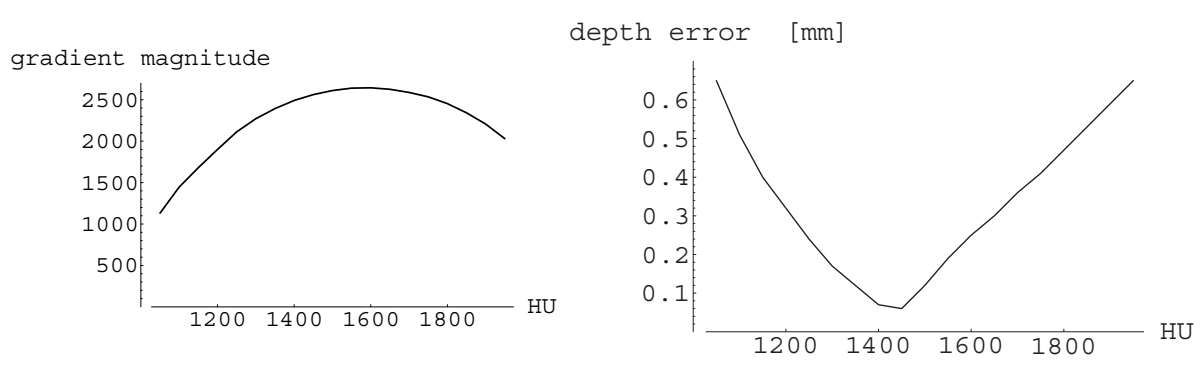

Fig. 4. Gradient magnitude (left) and localization error (right) for the simulated test object.
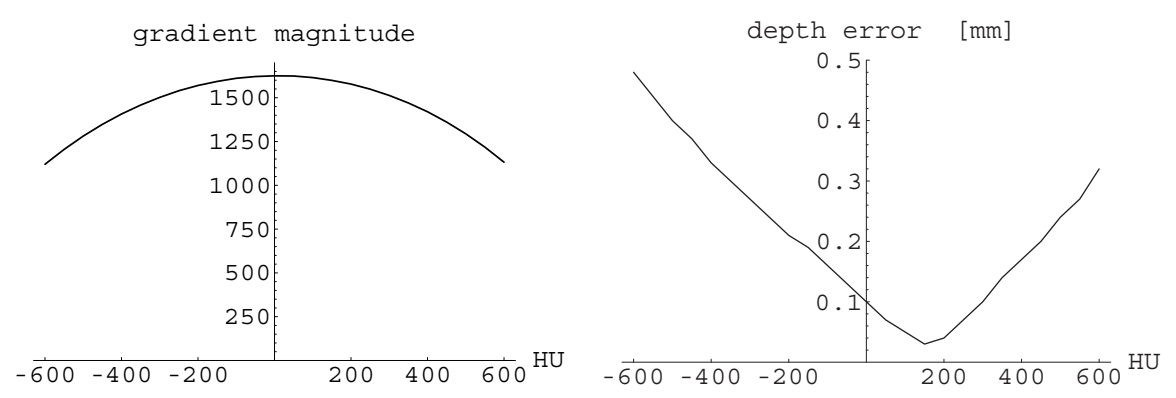

Fig. 5. Gradient magnitude (left) and localization error (right) for the teflon cone.

(measured of the still visible parts) seems little affected. This turns out to be due to the robust bisection algorithm.

Object Labels If only the label of the nearest voxel is considered at a sampling position, the underlying voxel grid becomes apparent. The algorithm presented in [7] chooses the most suitable label instead, based on the intensity at a sampling point. This way, these artifacts are completely eliminated.

\subsection{Variation of Segmentation Parameters}

Simulated Data Regardless of the other parameter settings, a poor threshold selection causes strong artifacts (fig. 3, bottom row), which also result in high localization errors. In this case, the lowest localization error is obtained for a threshold of about 1450, slightly below the $50 \%$ value (fig. 4), probably due to the small size of our test object. The gradient magnitude reaches its maximum at about 1550, yielding an error of less than $0.2 \mathrm{~mm}$. For a poor threshold, the error may be larger than the voxel size.

Phantom Data In this case, background and object intensities are in the approximate ranges of $-1000 \ldots-950$ and $900 \ldots 950$ Hounsfield units (HU) respectively, giving a 50\% threshold of about $-25 \mathrm{HU}$ and a localization error of about $0.1 \mathrm{~mm}$ (fig. [5). The gradient magnitude reaches its maximum at about the same value. Localization error is thus in the same range as for the simulated data. 


\section{Conclusions}

In this paper, we investigated to accuracy of isosurfaces in volume visualization, using simulated data and a phantom. With a suitable threshold selection, it could be shown that the accuracy is almost one order of magnitude better than the scanner resolution, and thus in the sub-mm range. However, acquisition and processing parameters have to be chosen carefully.

Determination of a suitable threshold value remains the most critical step. As could be shown, finding the maximum of the gradient magnitude seems a suitable method to get at least near a good threshold value. Whether this also holds for clinical data remains to be shown.

\section{Acknowledgement}

The CT dataset of the teflon cone is courtesy of Kornelius Kupczik, Evolutionary Anatomy Unit, University College London.

\section{References}

1. Bajaj, C. L., Pascucci, V., Schikore, D. R.: The contour spectrum. In Yagel, R., Hagen, H. (Eds.): Proc. IEEE Visualization '97. Phoenix, AZ, 1997, 167-173. (ISBN 0-8186-8262-0).

2. Dodgson, N. A.: Quadratic interpolation for image resampling. IEEE Trans. Image Process. 6, 9 (1997), 1322-1326.

3. Mitchell, D. P., Netravali, A. N.: Reconstruction filters in computer graphics. Comput. Graphics 22, 4 (1988), 221-228.

4. Pommert, A., Höhne, K. H.: Evaluation of Image Quality in Medical Volume Visualization: The State of the Art. In Dohi, T., others (Eds.): Medical Image Computing and ComputerAssisted Intervention, Proc. MICCAI 2002, Lecture Notes in Computer Science, SpringerVerlag, Berlin, 2002. (this volume).

5. Pommert, A., Tiede, U., Höhne, K. H.: Volume Visualization. In Toga, A. W., Mazziotta, J. C. (Eds.): Brain Mapping: The Methods, Academic Press, San Diego, CA, 2002, ch. 26, 707-723.

6. Tiede, U., Höhne, K. H., Bomans, M., Pommert, A., Riemer, M., Wiebecke, G.: Investigation of medical 3D-rendering algorithms. IEEE Comput. Graphics Appl. 10, 2 (1990), 41-53.

7. Tiede, U., Schiemann, T., Höhne, K. H.: High quality rendering of attributed volume data. In Ebert, D. et al. (Eds.): Proc. IEEE Visualization '98. Research Triangle Park, NC, 1998, 255-262. (ISBN 0-8186-9176-X).

8. Watt, A.: 3D Computer Graphics. 3. ed. Addison-Wesley, Reading, MA, 2000. 